\title{
Smart and Low Cost Technique for Forest Fire Detection using Wireless Sensor Network
}

\author{
Ahmad AA Alkhatib
}

\begin{abstract}
The line of sight and the early stage of the fire process problem could be solved with the second type of sensors. A new technology called Wireless Sensor Network (WSN) is nowadays receiving more attention and has started to be applied in forest fire detection. The Wireless nodes integrate on the same printed circuit board, the sensors, the data processing, the wireless transceiver and they all consume power from the same source- batteries. Unlike cell phones, WSN do not have the capability of periodic recharging. The sensors are devices capable of sensing their environment and computing data. The sensors sense physical parameters such as the temperature, pressure and humidity, as well as chemical parameters such as Carbon Monoxide, Carbon Dioxide and Nitrogen Dioxide. The sensors operate in a self-healing and self-organising wireless networking environment. One type of wireless technology is ZigBee which is a new industrial standard based on IEEE 802.15.4. This technology emphasises low cost battery powered application and small solar panels and is suited for low data rates and small range communications. Wireless Sensor Networks have seen rapid developments in a large number of applications. This kind of technology has the potential to be applied almost everywhere; this is why the research interest in sensor networks is becoming bigger and bigger every year.
\end{abstract}

Forest fire detection and prevention is another real problem faced by a number of countries. Different methods for monitoring the emergence of fires have been proposed. The early methods were based on manned observation towers but this technique was inefficient and not entirely effective. Subsequently, camera surveillance systems and satellite imaging technologies were tried but this also proved ineffective at being able to efficiently monitor the initial start of the surface fire. For example, camera networks can be installed in different positions in the forests but these provide only line of sight pictures and may be affected by weather conditions and/or physical obstacles.

The revolution of WSN technology in recent years has made it possible to apply this technology with a potential for early forest fire detection. These sensors need to be self- organised and follow an efficient algorithm, interfaced with other technologies or networks. A number of studies have considered using WSN in wood fire systems.
In this work, we relied on WSN and all nodes only use temperature sensors as they are programmed on a certain threshold temperature, above it the node will send an alarm message to the sink. This concept relies solely on the node behaviour to alert of crises possibility using simple node components to provide detection and information on whether this is a peaceful fire, or the beginning of wild fire. The key in this method is to make decisions by tracking the fire propagation and check the logic behind it.

\section{Keywords}

Wireless Sensor Network, WSN, efficient system for forest fire detection, efficient detection method for forest fire systems, Wireless Sensor Network for Forest Fire Detection and Decision Making

\section{INTRODUCTION}

Forest is playing an important role in the global, ecological, environmental and recreational system. It greatly impacts the amount of greenhouse gases, atmospheric carbon absorption, and reduces soil erosion. It can moderate the temperature, and regulate rainfall. To a large extent, Forest fires are among the most dangerous natural accidents that occur in practically all countries. Forest fire is a dominant disturbance factor in almost all the forest vegetation zones throughout the World, and it is considered to be a potential hazard causing physical, biological, and environmental consequences. Furthermore, forest fires can have adverse societal impacts regardless of whether they are caused by natural forces or human activities. One possible solution is to use Wireless sensor network technologies, which normally deploy a large number of small, low cost sensors, fairly densely that can observe and influence the physical world around them by gathering physical information, transform it into electrical signals, send it to a remote location to do some analysis and deploy the results in different applications. This means there is no need to build towers or set up complicated communication links such as; microwave and satellite. It can be deployed anywhere, even in inaccessible places. This technology can provide a real time monitoring for forest fire, where it can provide information at the ignition instance or at very small delay, depends on the node used wake up/sleep schedule. It's more reliable because it can influence the world in the surrounded area, if it is used in appropriate methods, rather than expecting events over large distances and long delay like other satellite and camera towers techniques.

\section{Research Objectives}

This research objective is to build a network through distributed wireless sensors randomly spread in the forest and to create a self-organized and robust network between the sensors to cover large areas in the forests that may be prone to/or in threat of fire damage at any time. 
The function of this sensor network will be to detect fire in the coverage area at regular intervals of time - say every 10-15 minutes- and to send a warning signal to a main server for further transmission to emergency services in the instant a fire is detected. When the fire is detected, all of the sensors in the area of vicinity will become active and commence the mandatory start and routing tasks. As the sensors are provided with small wireless range transmitters the data will be transmitted from sensor to sensor until the signal reaches the sink. When the sink receives the data it will start a processing routine and check if the fire clearly represents danger through a measure of the rate with which the fire spreads. If the result of processing is affirmative and real danger exists, then the sink determines the position of the fire. Then the sink will send an alarm signal to the fire department to include information about the exact location of the fire, the temperature, fire spread speed to understand the fire behaviour.

Based on the information received, the fire department will then be able to assess the extent and gravity of the situation to arrive at an optimal decision. In case of real danger, subsequent preventative work can then commence to mitigate the situation through appropriate action before the fire becomes uncontrollable. The main aim for this research can simply presented by the following picture:
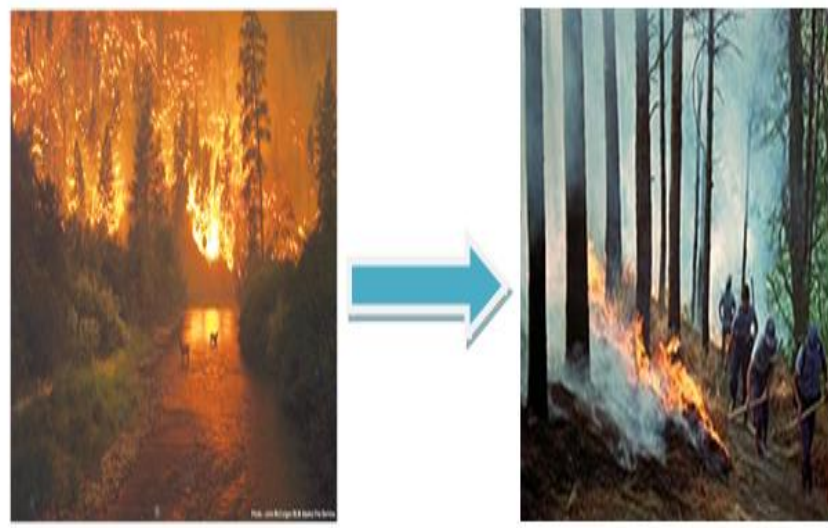

Figure 1: Research Aim

\section{Problem Description \& Previous Work}

Unfortunately, the forest fire is commonly observed only when it has already spread over a large area, making its control and stoppage arduous, and often even nearly impossible. The result is a devastating loss of lives (of firefighter crewmen and others) and property (valuable forest foliage and resources as well as clusters of houses and other buildings in the outlying areas). In addition, to irreparabledamage to the ecology (huge amount of smoke and carbondioxide ( $\mathrm{CO} 2)$ in the atmosphere). Among other terrible consequences of Forest Fire are such long-term disastrous effects as the impact on the local weather pattern; global warming; extinction of rare species of the flora and fauna; etc.

The problem with forest fires is that the forests are usually remote, abandoned/unmanaged areas full with trees, dry and parching wood, leaves....etc. that act as a fuel source. These elements form a highly combustible material and represent the perfect context for fire initial-fire ignition and as fuel for later stages of the fire. The fire ignition could be caused through human actions, like smoking or barbeque parties, or by natural reasons such as; high temperature in a hot summer day, or a broken glass working as a collective lens focusing the sun light on a small spot for a length of time thus leading to fireignition. Once ignition starts then the combustible material may easily fuel to feed the fire central spot. The spot then becomes bigger and wider. The initial stage of ignition is normally referred to as 'surface fire' stage. This may then lead to feed on adjoining trees and the fire flame becomes higher and higher thus becoming 'crown fire'. Mostly at this stage the fire becomes uncontrollable and damage to the landscape may become excessive that could last for a very long time depending on prevailing weather conditions and the terrain.

Millions of hectares of forest are destroyed by fire year after another. It is a large area and it produces more carbon monoxide than the overall automobile traffic. Monitoring of the potential risk areas and an early detection of fire can significantly shorten the reaction time and also reduce the potential damage as well as the cost of fire fighting. Known rule applies here: 1 minutes - 1 cup of water, 2 minutes - 100 litres of water, 10 minutes - 1000 litres of water. The objective is to detect the fire as faster as possible, its exact localization and early notification to the fire units

According to Buratiet al. (2009), a wireless sensor network (WSN) can be defined as "a network of devices, denoted as nodes, which can sense the environment and communicate the information gathered from the monitored field (e.g., an area or volume) through wireless links. The data is forwarded, possibly via multiple hops, to a sink (sometimes denoted as controller or monitor) that can use it locally or is connected to other networks (e.g., the Internet) through a gateway. The nodes can be stationary or moving. They can be aware of their location or not. They can be homogeneous or not"[1].

Forest fire detection and prevention is another real problem face a number of countries. Different methods for monitoring the emergence of fires have been initiated. The early methods were based on manned observation towers but this technique was inefficient and not entirely effective. Subsequently, camera surveillance systems and satellite imaging technologies were tried but this also proved ineffective at being able to efficiently monitor the initial start of the surface fire. For example camera networks can be installed in different positions in the forests but these provide only line of sight pictures and these may be affected by weather condition and/or physical obstacles. Satellite images have proved more efficient than camera surveillance, where images gathered by two satellites, the advanced very high resolution radiometer(AVHRR), launched in 1998 and the moderate resolution imaging spectro-radiometer (MODIS), launched in 1999 have been used[2]. Unfortunately, these satellites can provide images of the regions of the earth every two days and that is along time for fire scanning, besides the quality of satellite images can be affected by the weather condition

The revolution of WSN technology in recent years has made it possible to apply this technology with a potential for early forest fire detection. These sensors need to be self organised and follow an efficient algorithm, interfaced with other technologies or networks. A number of studies have considered using WSN in wood fire systems. Doolin et al [3] experimented with 10 sensors provided with GPS device, to sense temperature, humidity pressure and send these data back to the sink. The problem with this system is that the distance between sensors is too far (approximately $1 \mathrm{~km}$ ), in case of node failure a connection between some sensors and the sink may be lost and that could leave a gap in the network 
coverage. Lloret et al [4] suggested deploying a mesh network of sensors provided with internet protocol (IP) cameras. Here the sensors detect the fire at the beginning and send an alarm signal to the sink. The sink then sends a message to switch the cameras 'on' in the same area of the detected fire to provide real images of the fire at any time. Hartung et al [5] used WSN for wood fire detection as a hybrid with web cameras. The main target of their studies was to investigate the fire behaviour in forests. They used WSN to provide data for weather status and web cameras to provide the images of the fire.Son [6] proposes a project for fire detection in South Korea using Cameras surveillance hybrid with WSN. They propose a clustered topology for the network. Each cluster has a head node to do some calculations, for example; fire risk level by measuring temperature, humidity and some other parameters. In addition there is routing and data aggregation tasks included in their algorithm. In this method there is an increase in the power consumption rate in each head nodes, besides they do not consider the power balancing issue, which may result in some sensors deactivating before others thus leading to coverage gaps. Hafeeda et al [7] presents a very smart system. They base their network action on fire weather index (FWI). This index includes the probability of fire ignition and fire spread rate as well. FWI provides the moisture content in relation to weather observation where the fuel code describes the soil content of forest ground. See Figure 1.

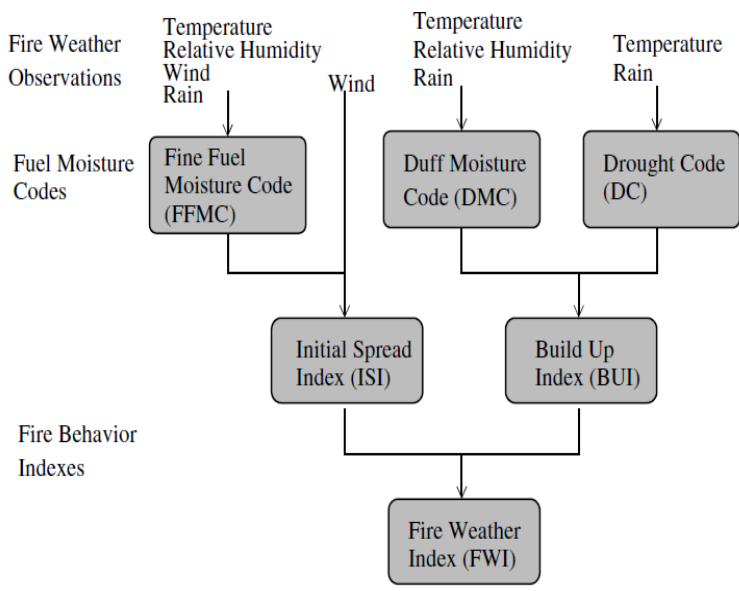

Figure 2: FWI System [7]

The previous methods can be summarised into four methods:

- The early methods were based on manned observation towers but this technique was inefficient and not entirely effective.

- Camera surveillance systems were tried, but this also proved an ineffective method for fire detection, regards to the need for manual instalment for each Camera in an appropriate position, rather than the line of sight images, night images and bad weather images problems.

- Satellite images gathered by two satellites[4], the advanced very high resolution radiometer (AVHRR), launched in 1998 and the moderate resolution imaging Spectroradiometer (MODIS), launched in 1999. These satellites can provide images of the regions of the earth every two days. However, this is a long delay for fire scanning, plus the quality of satellite images can be affected by the weather conditions. its impossible to deploy satellites to monitor all forest all the time.
- Finally, WSN started to be considering as a partial solution, where this kind of technology used with other technologies, such as IP cameras, weather databases and fuel databases.

The reason for making this research unique is because it has lower cost than other available systems. This network only uses the Wireless Sensor Networks without any other supporting technologies or databases. The nodes only provides with temperature sensors to detect the fire and the decision will based on the network behaviour.

\section{Background on forest fire}

Before jumping directly into a discussion of wildfire and its behaviour, let's provide a definition for fire forest[8]:

Combustion is a complex process in which fuel is heated, ignites, and oxidizes rapidly, giving off heat in the process. Fire is a special case of combustion-self-perpetuating combustion characterized by the emission of heat and accompanied by flame and/or smoke. With fire, the supply of combustible fuel is controlled by heat given off during combustion(Scott 2012).

Maintaining a fire requires three factors that must be presented. If any one factor is missing, the fire will go out. There must be a source of fuel available for combustion, a source of heat to promote the reaction (the fire itself), and oxygen in sufficient concentration to maintain the reaction [8].

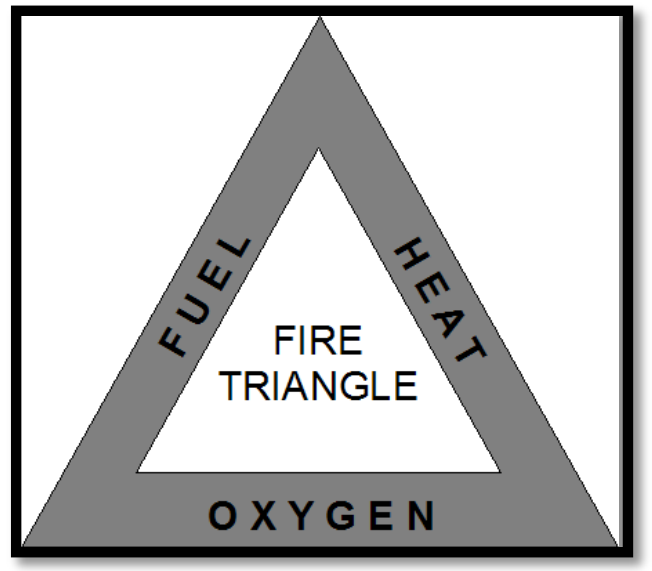

Figure 3: Fire Factors Triangle[8]

A forest fire is generally a dynamic phenomenon that changes its properties and behaviour from one place to another and with the passage of time. Owing to the fact that the forest fuel available in a given location is limited, for a fire to continue it must spread to neighbouring fuel. This is performed through the complex heat-transfer and thermochemical processes that determine fire behaviour [9].

Describing wildfire morphology by identifying the parts of a fire is very helpful during wildfire operations (as they can have significant effects on fire fighter safety).Predicting wildfire behaviour is as much an art as it is a science and very much based on understanding weather conditions that influence wildfire. Even seasoned fire fighters have trouble reading fire behaviour and in predicting a forest fire's potential threat to property and lives.

A wildfire burning in a constant environment takes the shape of an ellipse. The fire environment can be variable within time once a fire grows beyond the ignition area. A fire might have different parts burning in different environments, such as wind direction, slope, moisture contents, wind speed, etc. This heterogeneity of fire environment might have resulted in a 
very complex shape, even if each part of the fire spreads in ellipse shape[8,10].

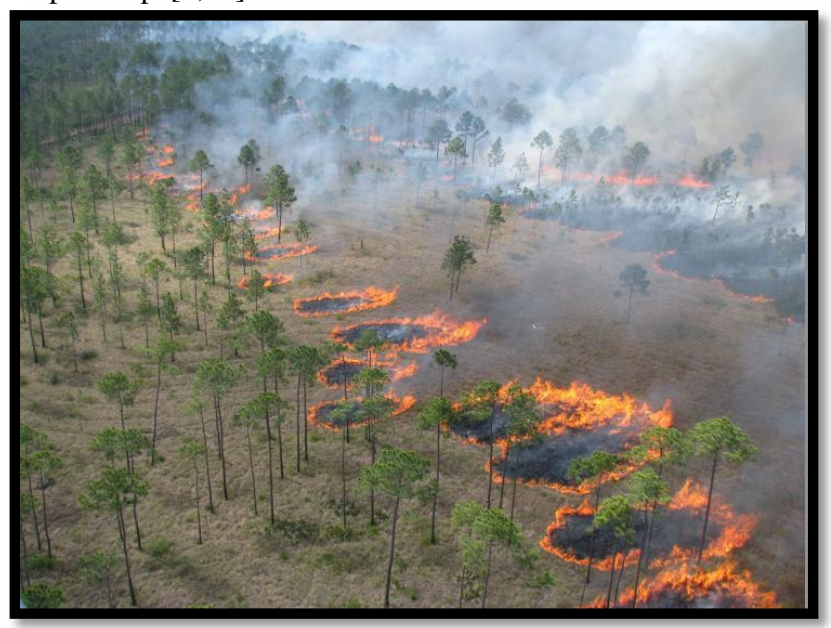

Figure4: simple ellipse shape for fire under constant fire environment[8]

Different parts of the fire are as follows:[8]:

- A finger is a long, narrow extension of the main body of fire.

- A pocket is an unburned indentation of the fire perimeter surrounded on three sides by the fire.

- An island is an unburned area within a fire that is wholly surrounded by burned area.

- A spot fire is a fire ignited outside the main fire by a firebrand.

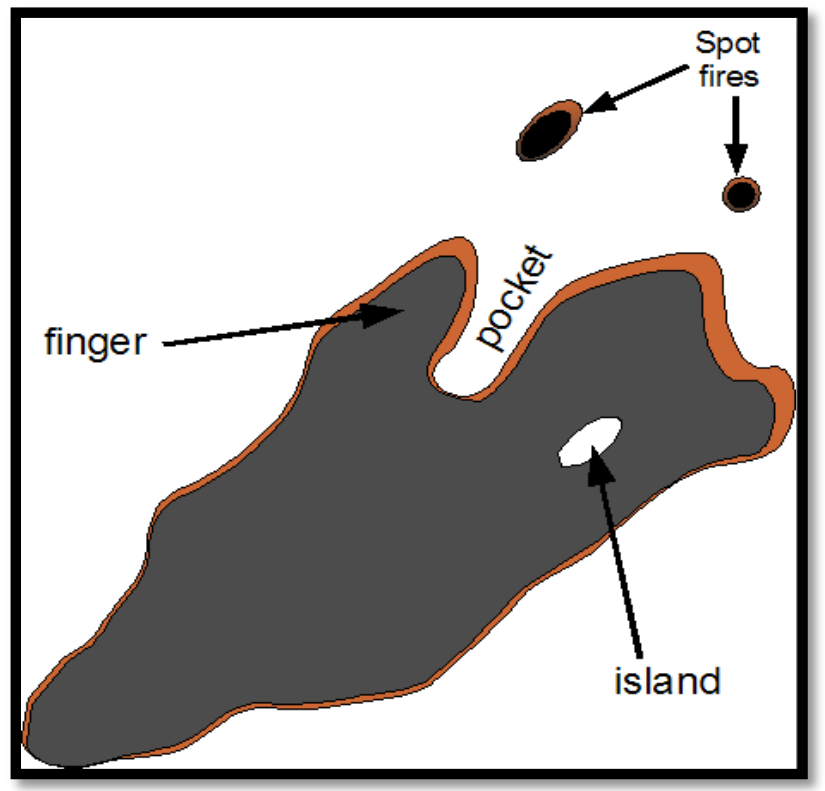

Figure 5: Fire different parts[8]

These parts can occur at many different scales. For example, a finger can stretch over a few feet or more than a mile. The fire might have fingers on fingers and spot fires from spot fires.

Since the fire shape under constant environment is ellipse, the long axis of the ellipse is the direction of the maximum fire spread, as shown in the following figure. Where, the relative spread direction is the angle between the front flam orientation and the direction of maximum spread, measured in degrees clockwise from the direction of maximum spread[8,9].

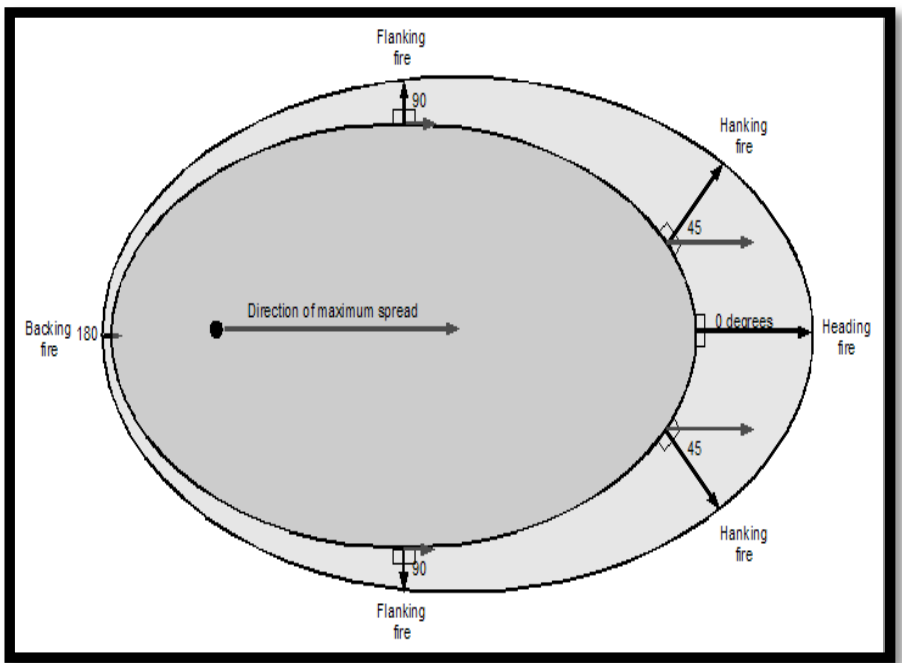

Figure 6: wildfire spread under constant environment[8] In a heterogeneous fire environment, the direction of maximum spread can be different in the fire various areas. Each area has its own environment and therefore its own direction of maximum spread. But no matter how complex a fire is, by examining the orientation of the flaming fire front with respect to the direction of maximum spread (heading direction; shown by arrow), wildfire morphology by relative spread direction can be related to fire shape. $[10,12]$

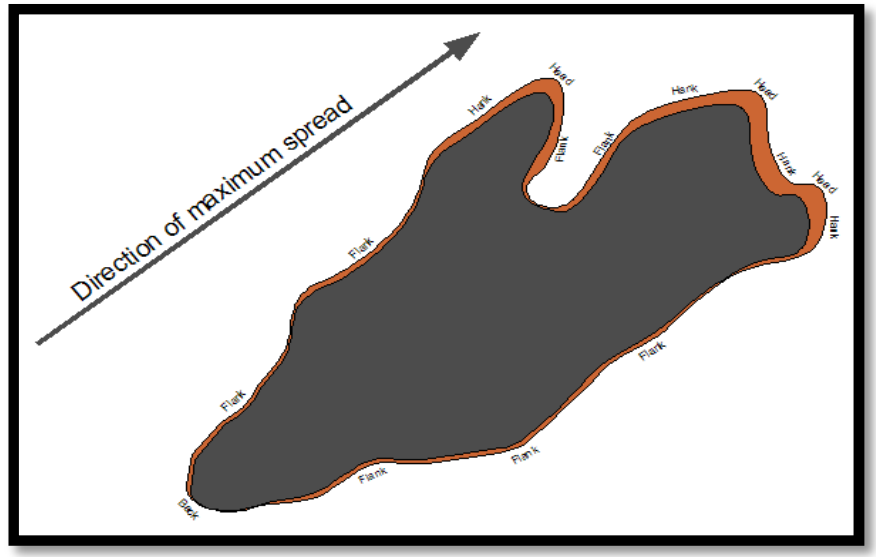

Figure 7: Fire spread under heterogeneous environment[8]

Three main regimes of fire propagation: ground fire; surface fire and crown fire. Forests are the perfect fire environment, typical of its shrubs and small trees, resulting in a low canopy base height and making crown fire initiation a common occurrence. A crown fire occurs when surface fire grows vertically and reaches the tree crowns. At this stage, the fire will start to propagate as a dangerous crown fire, which is hard to extinguish[9]

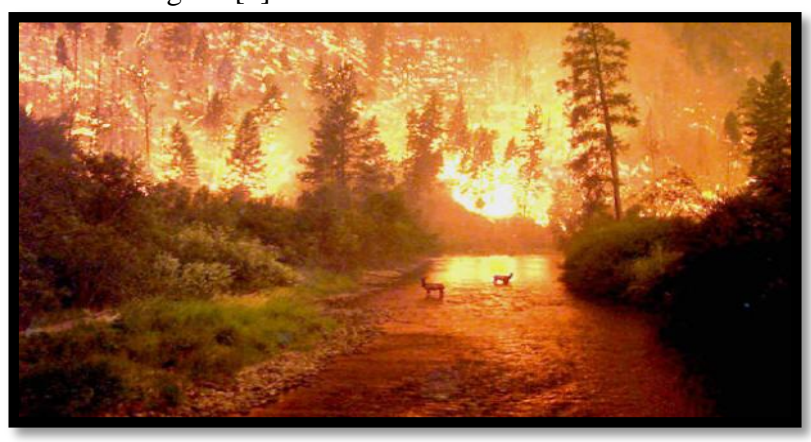

Figure 8: Crown Fire 


\section{Fire detection and decision making}

The use of sensors to detect and monitor fire behaviour has enhanced the application of new technologies in the fire field. Sensors are able to consider certain dynamic and static variables such as humidity, the type of fuel, slope of the land, the direction and the speed of the wind, smoke, etc. They allow us to determine the direction and possible evolution of the flame front. The sensor-based systems can be very useful to detect a fire and to take decisions to eradicate it.

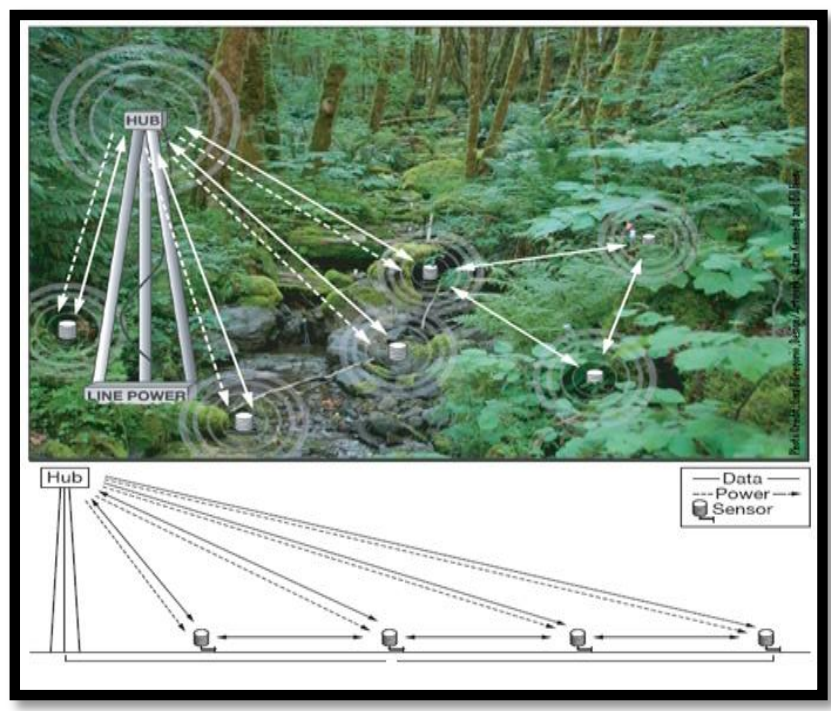

Figure 9: Sensor Network for fire detection

The previous work relied on images or databases such as Weather index, Fuel Index models, gas boards and intelligent sensors to make the decision. In this research, all nodes have known location, and nodes only use temperature sensors and they are programmed on a certain threshold temperature, above it the node will send an alarm message to the sink. This concept relies solely on the node behaviour to alert of crises possibility using simple node components to provide detection and information on whether this is a peaceful fire, or the beginning of wild fire. The key in this method is to make decisions by tracking the fire propagation and check the logic behind it rather than using complicated databases or imaging technologies. The most convenient method is to monitor forests by using a GUI to represent the events and alerting messages on the monitoring screen using some logical evaluation to come up with a decision. 50 nodes had been tested in NS2 with multiple scenarios, see the following figure8.

Once one of the nodes detect the fire all nodes in range of \pm 45 degrees wake up and start working as a router and a sensor, if they can do any sensing at that stage, every minute to track the

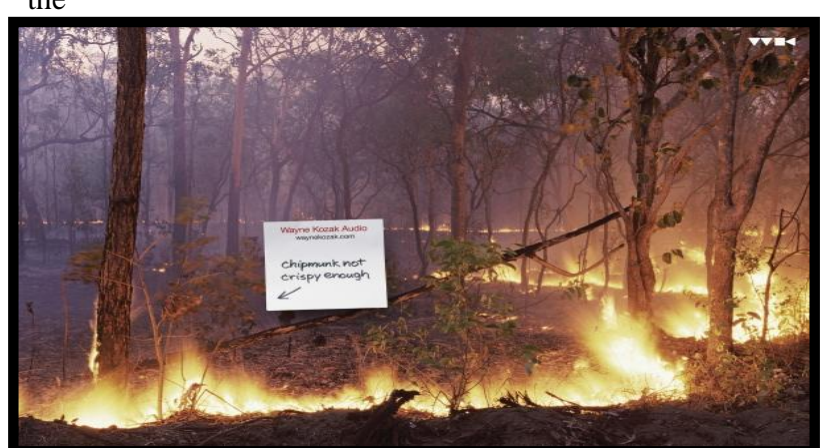

Figure 10: Medium propagation fire

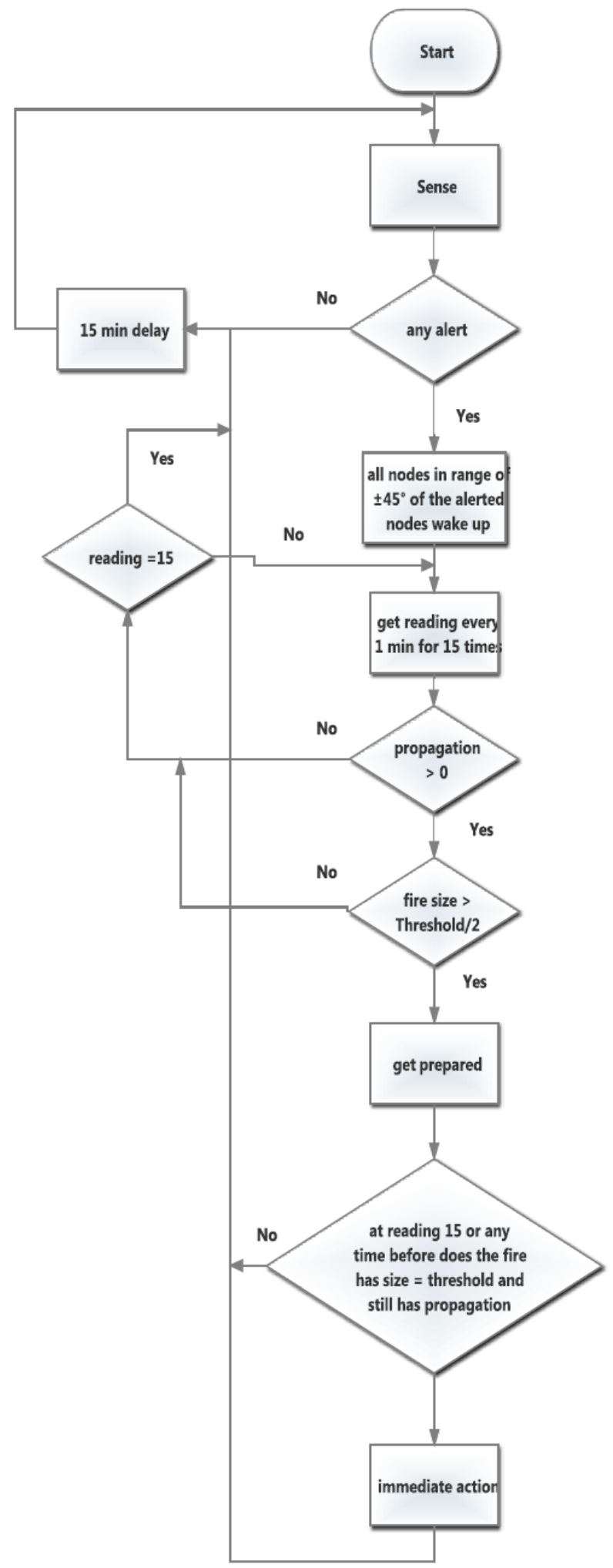

Figure 11: fire detection method flowchart 
As it appears in the above picture, the fire is propagating over a large distance, and is small in width with low fire density. This type is very easy to detect because its going to increase the temperature in the area, and it propagates over large a distance and it's going across many sensor ranges.

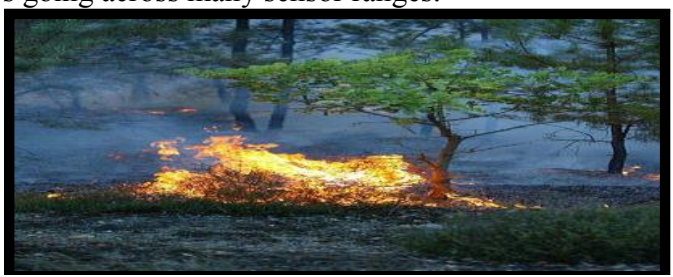

Figure 12: Slow propagation fire

This type is kind of a tricky one, it might be extinguished on its own and there is no need for any action because the fire growth is very slow and has stopped at a certain time. On the other hand it might grow to become a danger; in that case the nodes keep track of the fire growth until it's larger than the threshold value.

$>$ Case 3: very hot sunny day, there might be a rise in the temperature over the threshold value, since it's a sunny day, the temperature will be almost the same everywhere and its impossible all the sensors or group of sensors start to provide alarms at the same time, in this case the threshold value must be increased to a more suitable value.

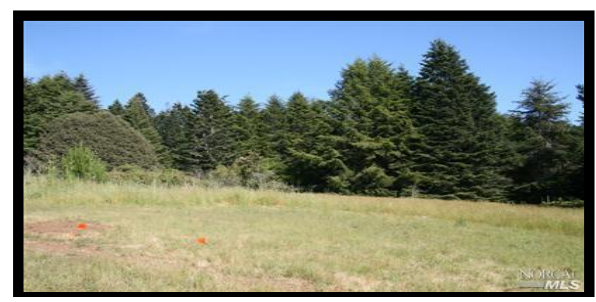

Figure 13: Sunny day in the forest

During hot days the sensors might be under the trees or directly in the sun light. So some sensors might give alarm and others not, but the propagation is not logical. The alarm will come from many different discrete nodes at the same time, which is not indicating any propagation or growth for fire.

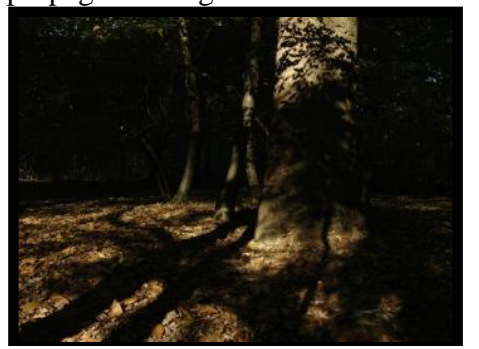

Figure 12: Sensors in sun light and shadow

$>$ Case 4: cold days in forests have less possibility for fires, so the temperature threshold might be reduced to a suitable value.

Relation between the sensor deployment scheme and early detection: When the early detection goal is considered, again regular and moderate density deployment schemes are more successful. The average distance between a fire ignition location and the closest sensor node decreases when the density in random deployment case increases. This means it will take less time until a sensor node detects the increase in temperature due to a fire igniting. Additionally, as the distribution among the sensor nodes provides a difference in the sensing performance. Also, when the number of nodes increases, fire detection is much faster because of the overlap coverage and short gaps between the sensing ranges.
The distance between the sensor nodes affects the time required for the heat waves to reach to the sensor nodes. It is observed that it might takes more than 10 minutes for the sensor nodes to sense the fire thread when the distance between fire ignition location and the sensor node gets more than 20 meters. This experiment shows that the distance between the sensor nodes should be kept less than 20 meters for a successful early detection system and this is what is applied in the coverage method.

Environment-aware scheme's performance is much better in terms of this metric. During the months that are not in the fire season, the fire threat level is low, so the period of sleep time can be longer. However, in fire-season the average fire detection time of the environment aware model is too short since the fire threat during that period is taken into account.

By using the sensing result, simple information can be provided for the fire fighters about the fire behaviour, such as ignition point, fire spread speed, direction of maximum spread. The simple simulation result for fire behaviour can help in fire fighter safety and fire extinguishing. The team work of the fire fighter can be organised according to these results, for example, if the fire spreads toward a human inhabited area, or very precious properties, the team target is to stop the spreading in that direction, so more fire fighters can be deployed in the front flame of the spread direction. On the contrary, if the fire needs to be distinguished as soon as possible, more fire fighters can be deployed in the back of the fire, to weak the front flame, reduce the fire intensity, limit the spreading, reduce the danger and make it easier to extinguish.

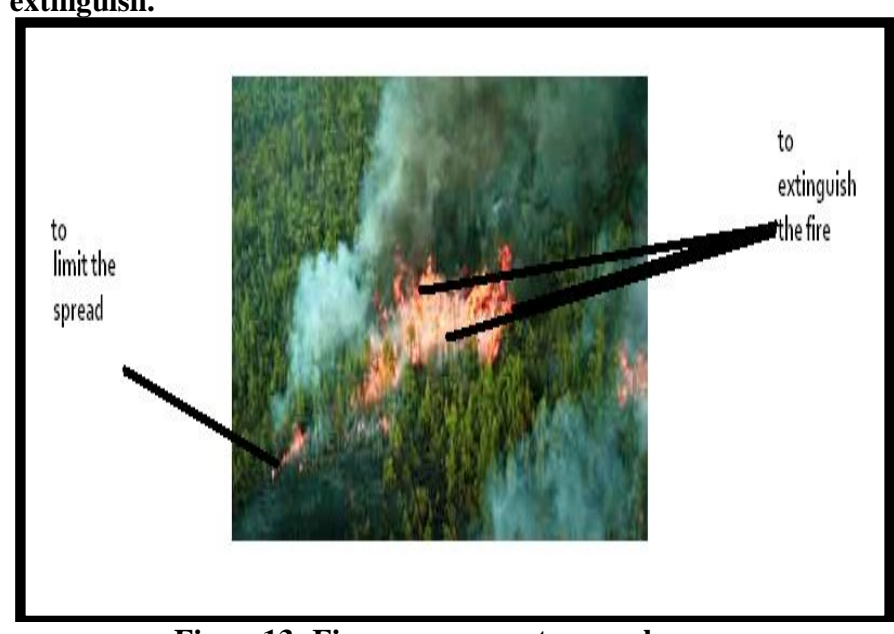

\section{Acknowledgment}

Our thanks to the experts who have contributed towards development of this paper.

\section{Conclusion}

Fire detection and decision making in this wok can provide some advantages:

- $\quad$ No need for complicated gas boards and specialized devices or connect the network to data basses and applying complicated models to detect fires. Only simple cheap temperature sensor required on each node.

- Helps in decision making by distinguishing between peaceful fire, fault alarms and potential danger require immediate reaction.

- Low possibility of false alarms.

- Provide some information about the fire behaviour for fire fighters to help in team work organising. 


\section{References}

[1] Buratti, C., et al., An Overview on Wireless Sensor Networks Technology and Evolution. Sensors, 2009. 9(9): p. 6869-6896.

[2] The Advanced Very High Resolution Radiometer (AVHRR) [cited jul/ 2011]; Available from: Http://noaasis.noaa.gov/noaasis/ml/avhrr.html/.

[3] Doolin, D., M., Sitar, N., Wireless Sensor Nodes for Wildfire Monitoring in SPIE Symposium on Smart Structures and Materials. 2006: San Diego.

[4] Lloret, j., Garcia, M., Bri, D., Sandra, S., A Wireless Sensor Network Deployment for Rural and Fire Dtetection and Verification. Sendor Nodes, 2009. 9(11): p. 8722-8747.

[5] Hartung, C., Han, R., FireWxNet: A Multi-tiered Portable Wireless System for Monitoring Weather Conditions in Wildland Fire Environments, in 4th International Conference of Mobile Systems, Applications and Services. 2006. p. 28-41.
[6] Son, B., A Design and Implimintation of forest fires surveillance System Based on Wireless Sensor Networks for South Korea Mountains. International Jornal of Computer Science and Network Security, 2006. 6: p. 124-130.

[7] Hafeeda, M., Bagheri, Forest Fire Modling and Early Detection Using Wireless Sensor Networks. Ad Hoc Sensor Wireless Networks, 2009. 7: p. 169-224.

[8] Scott, J. H. (2012). Introduction to Wildfire Behaviour Modeling. National Interagency Fules, Fire, \&Vegetation Technology Transfer., Wild Fire Managment RD\&A: 7-15.

[9] Viegas, D. (1993). "Fire Behaviour and Fireline Safety."Annual Maditerranean Burns Club6(3).

[10] Finney, M. A. (2004). FARSITE: Fire Area SimulaterModel Development and Evaluation. f. s. US department for agriculture, USDA: research parer RMRS-RP-4 Revised 